Published in "Nature Nanotechnology 15(9): 729-730, 2020" which should be cited to refer to this work.

\title{
When plants and plastic interact
}

Overcoming the challenges of plastic detection in plants has made it possible to transfer many of the lessons learned from plant-metal nanoparticle interactions to plastic nanoparticles.

\section{Fabienne Schwab, Barbara Rothen-Rutishauser and Alke Petri-Fink}

$\mathrm{P}$ lants have always interacted with, and evolved in the presence of, nanoparticles from natural sources such as weathering processes in soils and surface waters or erupting volcanoes ${ }^{1}$. Human awareness of nanoparticleplant interactions rose about a decade $\mathrm{ago}^{2}$ with the increasing environmental exposure of anthropogenic incidental or engineered nanoparticles. Recent research on these interactions has led to exciting progress, most notably in the field of nanoagrochemicals ${ }^{3,4}$. With the emergence of plastic nanoparticles, or nanoplastics, in the environment due to human activities such as littering, use of plastic textiles and personal care products ${ }^{5}$, and most recently, polymer-based nanoagrochemicals, the interactions of plastic nanoparticles with plants have caught the interest of scientists ${ }^{6}$.

In contrast to animal cells, plant cells can accumulate only a small fraction of intact nanoparticles in their intracellular compartments known as symplast (Fig. 1). The limited uptake is mainly due to the fact that plants possess, in addition to the nanometre-thin phospholipid bilayer cell membrane, a strong cellulose cell wall and a coextensive pectin network that can be up to several micrometres thick (Fig. 1). Along and in between these cell walls, nanoparticles, especially those that are negatively charged, can move to some extent in microscopic extracellular channels and reach the vasculature responsible for water transport, which is called apoplastic transport. Once within the water transport system, nanoparticles can rapidly translocate into the stem, leaves and potentially also the fruits; albeit further barriers will hinder the accumulation of the nanoparticles in the latter reproductive organs ${ }^{2}$. Mucilage and exudates are less known but equally important barriers for nanoparticles, especially on root tips, root hairs and stomata (pores in leaves for gas exchange). For unicellular algae, excess mucilage production in response to nanoparticle exposure is a common phenomenon ${ }^{2}$. In higher plants, mucilage and exudates act as a negatively charged first layer of protection and have been reported to block positively charged metal nanoparticle uptake ${ }^{7}$ on the outer edge of the cell wall (Fig. 1), for example in the root-growth medium interface (rhizosphere).

Our knowledge of nanoparticle movements in plants mostly originates from experiments on metal and metal oxide nanoparticle-plant interactions. Determining the fate of plastic nanoparticles in plants is much more challenging because plant tissue is essentially a complex mix of biopolymers and the detection of plastic in this environment is challenging. Now, Sun et al. use labelled particles with different surface charges to show that processes similar to those of relatively inert metal or metal oxide nanoparticles occur in the roots for polystyrene plastic nanoparticles.
Negatively and positively charged plastic nanoparticles move similarly in roots of Arabidopsis thaliana (thale cress) ${ }^{6}$ when compared to, for example, negatively charged gold nanoparticles investigated earlier ${ }^{7}$. By combining electron microscopy, confocal fluorescence microscopy and different plastic nanoparticles labelled by either metals or fluorescent dyes, Sun et al. demonstrate that both particle types accumulate to some extent on the root surface, but the negatively charged plastic nanoparticles can, in contrast to the positively charged particles, reach the plant vasculature. From there, they can travel widely within the plant as already shown earlier ${ }^{2,6}$. The positively charged plastic nanoparticles, likely due to electrostatic interactions, aggregate within plant exudates, or end up stuck in areas where a negatively charged protective mucilage layer is present such as the root border cells and root hairs, and thus mainly adsorb on the plant surface.

Sun et al. also examined the toxicological effects of the plastic nanoparticles on the plants at relatively high concentrations in the $\mathrm{g} \mathrm{kg}^{-1}$ and $\mathrm{mg}$ $1^{-1}$ range. The resulting moderate reduction in growth was less pronounced in soil than in culture medium, highlighting a mediating effect of soil on the toxicity or uptake. Exposure to the positively charged plastic nanoparticles triggered mild plant defence reactions; as reflected by an 


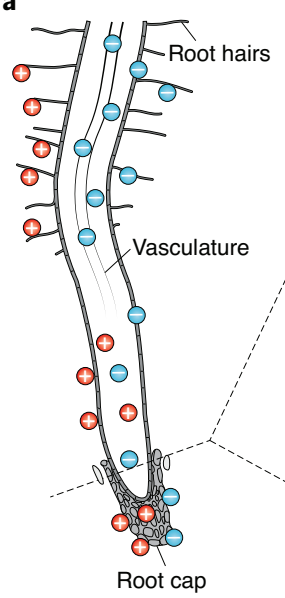

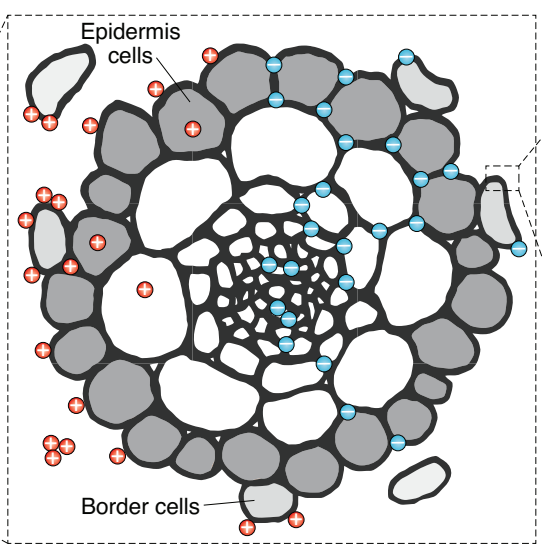

c

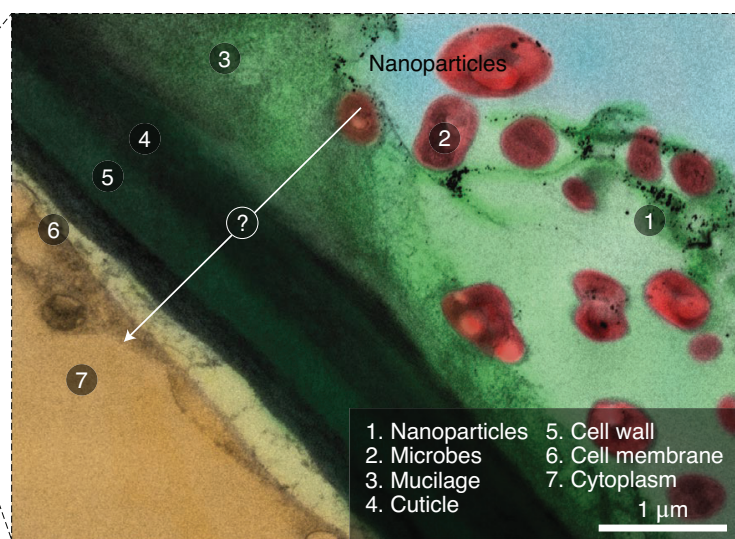

Fig. 1 | A plant root interacting with nanoparticles, such as plastic nanoparticles. a, Sketch of a root long section showing interactions with positively (red) and negatively (blue) charged nanoparticles. b. Zooming into a root tip cross-section shows the apoplastic and symplastic uptake of negatively and

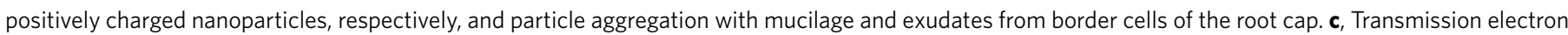
micrograph showing the different defensive barriers of the plant cell wall in false colours; the outside of the plant in soft blue: (1) Electron-dense dark spots are intact gold nanoparticles $15 \mathrm{~nm}$ in diameter; (2) Symbiotic or parasitic microbes in the root-soil interface that can affect nanoparticle degradation; (3) Mucilage consisting of gel-like high molecular weight exudates act as a strong barrier for nanoparticles and, apparently, plastic nanoparticles; (4) Cuticle layering the outer cell wall; (5) The cellulose plant cell wall, the main reason for the high impermeability of plants to nanoparticles; (6) The cell membrane

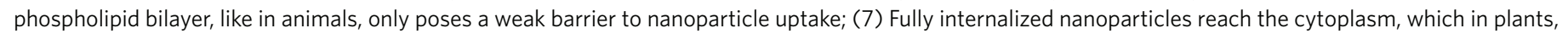
together with the cell membrane, belongs to the symplast where cell-to-cell transport occurs through channels between cells called plasmodesmata with basal size exclusion limits of $3-4 \mathrm{~nm}$.

increased production of exudates (oxalate) trapping the particles in aggregates, and elevated stress in the roots. For the negatively charged plastic nanoparticles, the biochemical responses of the plants were less pronounced. This indicates that primarily the adsorbed positively charged plastic nanoparticles, or a small fraction of intracellular positively charged particles, can cause some toxicity and corresponding defence responses, which agrees with earlier work on metal and metal oxide nanoparticles ${ }^{2,7}$. Future research will need to address whether the internalized or the adsorbed fraction of plastic nanoparticles is responsible for the defence reactions of the plant, which size fractions can accumulate, what the roles of high molecular weight mucilage molecules are and how the uptake behaviour of laboratory-synthesized plastic nanoparticles compares to more heterogeneous and non-spherical naturally weathered particles.

The rigorous study of Sun et al. provides a first insight into how plastic nanoparticles interact with terrestrial plants, specifically on negatively and positively charged plastic nanoparticles and their transport routes to the vasculature. It also shows the challenge of detecting plastic nanoparticles - that is, polymer particles - within a complex plant biopolymer matrix, and how this could be overcome by a combination of heavy metal and dye-labelled particles. Both labels used alone can lead to artefacts mostly related to particle degradation or leaching, such as toxicity or false-positive and negative uptake ${ }^{2}$. Sun et al. have addressed these experimental challenges by using different particles with either detection system in parallel, and by rigorous purification and leachate analysis of the particles before the experiments. The mechanisms Sun et al. report show that particle charge can influence the interaction with plants and induction of effects of plastic nanoparticles. However, the relatively moderate responses, especially in soil - despite high exposure concentrations and observed uptake indicate that plants had evolved interaction mechanisms with natural nanoparticles a long time before they encountered plastic nanoparticles, and now can activate ancient mechanisms to cope with them.
Fabienne Schwab (D) 1 , Barbara Rothen-Rutishauser (D) 1 and Alke Petri-Fink ${ }^{1,2}$

${ }^{1}$ Adolphe Merkle Institute, University of Fribourg, Fribourg, Switzerland. ${ }^{2}$ Chemistry Department, University of Fribourg, Fribourg, Switzerland.

$\bigotimes_{\text {e-mail:fabienne.schwab@alumni.ethz.ch }}$

1. Hochella, M. F. Jr. et al. Science 363, eaau8299 (2019).

2. Schwab, F. et al. Nanotoxicology 10, 257-278 (2016).

3. Wang, P., Lombi, E., Zhao, F.-J. \& Kopittke, P. M. Trends Plant Sci. 8, 699-712 (2016)

4. White, J. C. \& Gardea-Torresdey, J. Nat. Nanotechnol. 13, 627-629 (2018)

5. Lehner, R., Weder, C., Petri-Fink, A. \& Rothen-Rutishauser, B. Environ. Sci. Technol. 53, 1748-1765 (2019).

6. Sun, X.-D. et al. Nat. Nanotechnol. https://doi.org/10.1038/ s41565-020-0707-4 (2020).

7. Avellan, A. et al. Environ. Sci. Technol. 51, 8682-8691 (2017).

\section{Competing interests}

F.S. was supported by the Swiss National Science Foundation (project no. 168187) and Innosuisse (project no. 38515.1 IP-EE). All authors were supported by the Adolphe Merkle Foundation. 